\title{
On Localized Weak Precompactness in Banach Spaces
}

\author{
By \\ Minoru MATSUDA*
}

\begin{abstract}
In this paper we make a study of $K^{-}$-weakly precompact sets $A$ in Banach spaces. We give various characterizations of such sets by the effective use of the lifting theory, weak ${ }^{*} \bar{A}^{*}$-dentability and a $K^{-}$-valued weak ${ }^{*}$-measurable function constructed in the case where $A$ is non- $K$-weakly precompact. These results also can be regarded as generalizations of corresponding ones on Pettis sets and weakly precompact sets.
\end{abstract}

\section{\$1. Introduction}

Throughout this paper, $X$ denotes an arbitrary real Banach space, $X^{*}$ the topological dual space of $X$, and $B(X)$ the closed unit ball of $X$. The triple ( $I$, $\Lambda, \lambda)$ refers to the Lebesgue measure space on $I(=[0,1]), \Lambda^{+}$to the sets in $\Lambda$ with positive measure, $L_{1}$ to $L_{1}(I, \Lambda, \lambda)$ and $L_{\infty}$ to $L_{\infty}(I, \Lambda, \lambda)$. For each $E \in$ $\Lambda^{+}$, denote $\Delta_{E}=\left\{\chi_{F} / \lambda(F): F \subset E, F \in \Lambda^{+}\right\}$. We always understand that $I$ is endowed with $\Lambda$ and $\lambda$. If $C$ is a subset of $X^{* *}$, a function $f: I \rightarrow X^{*}$ is said to be $C$-measurable if the real-valued function $\left(x^{* *}, f(t)\right)$ is $\lambda$-measurable for each $x^{* *} \in C$. Especially, if $C=X$ (resp. $C=X^{* *}$ ), we say that $f$ is weak*-measurable (resp. weakly measurable). A function $f: I \rightarrow X^{*}$ is said to be weak*-scalarly null if $(x, f(t))=0 \lambda$-a.e. on $I$ for every $x \in X$. We say that a function $f: I \rightarrow X^{*}$ is weak*-equivalent to a $C$-measurable function $g: I \rightarrow$ $X^{*}$ if $f-g$ is weak*-scalarly null. If $f: I \rightarrow X^{*}$ is a weak ${ }^{*}$-measurable function with bounded range, we obtain a bounded linear operator $T_{f}: X \rightarrow L_{1}$ given by $T_{f}(x)=x \circ f$ for every $x \in X$, where $(x \circ f)(t)=(x, f(t))$ for every $t \in I$. Then the dual operator of $T_{f}$, denoted by $T_{f}^{*}\left(: L_{\infty} \rightarrow X^{*}\right)$, can be extended $\mathrm{u}$ niquely so as to be a bounded linear operator $S_{f}: L_{1} \rightarrow X^{*}$. A bounded linear operator $T: L_{1} \rightarrow X$ is said to be Dunford-Pettis (or completely continuous) if $T$ carries weakly convergent sequences of $L_{1}$ onto norm convergent sequences of

Communicated by T. Kawai, October 5, 1995.

1991 Mathematics Subject Classification: 46B07, 46B20, 46B22, 46 G10.

* Department of Mathematics, Faculty of Science, Shizuoka University, Ohya, Shizuoka 422, Japan. 
$X$. Note that a bounded linear operator $T: L_{1} \rightarrow X$ is Dunford-Pettis if and only if $\left\{T\left(\chi_{E}\right): E \in \Lambda\right\}$ is relatively norm compact.

Let $Y$ be a compact Hausdorff space. Then $f: Y \rightarrow X^{*}$ is said to be universally scalarly measurable if for each $x^{* *} \in X^{* *}$ the real-valued function $(x * *$, $f(y))$ is measurable with respect to all Radon measures on $Y$. A closed bounded convex subset $C$ of $X$ is said to be a weak Radon-Nikodym set if every bounded linear operator $T: L_{1} \rightarrow X$ such that $T\left(\Delta_{I}\right) \subset C$ can be represented by a Pettis kernel $f$ taking its value in $C$. As a general notion of weak*-compact convex weak Radon-Nikodym sets of $X^{*}$, the notion of Pettis sets is defined ([22]). From now on, we always understand that for each weak*-compact subset $H$ of $X^{*}, H$ is equipped with the weak*-topology $\sigma\left(X^{*}, X\right)$.

Definition 1. A weak*-compact (not necessarily convex) subset $K$ of $X^{*}$ is called a Pettis set if the identity map $i: K \rightarrow X^{*}$ is universally scalarly measurable.

It has been shown in [22] (or [16]) that $K$ is a Pettis set if and only if $\overline{c o}^{*}(K)$ (: the weak*-closed convex hull of $K$ ) is a weak Radon-Nikodym set if and only if every sequence $\left\{x_{n}\right\}_{n \geq 1}$ in $B(X)$ has a pointwise convergent subsequence $\left\{x_{n(k)}\right\}_{k \geq 1}$ on $K$ (that is, for every $x^{*} \in K, \lim _{k \rightarrow \infty}\left(x_{n(k)}, x^{*}\right)$ exists). We then define the notion of localized weak precompactness in Banach spaces as follows (see [8] and [2]).

Definition 2. Let $A$ be a bounded subset of $X$ and $K$ a weak ${ }^{*}$-compact subset of $X^{*}$. Then we say that $A$ is $K$-weakly precompact (or, $A$ is weakly precompact with respect to $K$ ) if every sequence $\left\{x_{n}\right\}_{n \geq 1}$ in $A$ has a pointwise convergent subsequence $\left\{x_{n(k)}\right\}_{k \geq 1}$ on $K$.

Note that if $K=B\left(X^{*}\right), A$ is simply said to be weakly precompact, which has been considered in [17] to characterize Banach spaces not containing a copy of $l_{1}$. We also know from the characterization of Pettis sets stated above that for each weak*-compact subset $K$ of $X^{*}, K$ is a Pettis set if and only if $B(X)$ is $K$-weakly precompact. Further, if $C(K)$ is the Banach space of all real-valued continuous functions on $K$ and $e: X \rightarrow C(K)$ is the natural point evaluation map (i.e. $e(x)\left(x^{*}\right)=\left(x, x^{*}\right)$ ), then it is easy to see that $A$ is $K^{-}$weakly precompact if and only if $e(A)$ is weakly precompact in $C(K)$.

In [2], Bator and Lewis have made a systematic study of localized weak precompactness and obtained its various characterizations which are analogous results of Fitzpatrick [3], Saab [20] and Saab and Saab [21]. Well, in a series of our papers [10], [11], [12], [13] and [14], we have obtained a number of characterizations of Pettis sets with the help of our function in [10]. We then have noticed that this $K$-valued weak ${ }^{*}$-measurable function constructed in the 
case where $K$ is a non-Pettis set plays very important role in every occasion. Therefore, when $A$ is non- $K$-weakly precompact, we think that it is important to construct a function which plays the same role as in the case where $K$ is a non-Pettis set. In fact, this can be done in the following by the same way as in $[10]$.

On the other hand, we became aware of the availability of a similar approach to analyze localized weak precompactness (especially, Pettis sets), seeing an approach due to Girardi and Uhl [6] to give a simpler proof of the fact that dentability implies the Radon-Nikodym property. To adopt such an approach, we need the following definitions, which have been introduced in [2] .

Definition 3. Let $C$ be a subset of $X^{* *}$. A weak*-compact subset $K$ of $X^{*}$ is said to have the $C$-point of continuity property $(C-P C P)$ if for every nonempty weak*-compact subset $M$ of $K$, the restriction of every $x^{* *} \in C$ to $M$ has a point of continuity. If $K$ has the $B\left(X^{* *}\right)-P C P$, then $K$ is said to have the scalar point of continuity property.

Definition 4. Let $K$ be a bounded subset of $X^{*}$. A weak-open slice of $K$ is a set of the form:

$$
S(x, \alpha, K)=\left\{x^{*} \in K:\left(x, x^{*}\right)>\sup _{y^{*} \in K}\left(x, y^{*}\right)-\alpha\right\}
$$

where $x \in X$ and $\alpha>0$.

Let $C$ be a subset of $X^{* *}$. Then the set $K$ is said to be weak-C-dentable, if for every $\varepsilon>0$ and $x^{* *} \in C$, there exists a weak*-open slice $S$ of $K$ such that $0\left(x^{* *} \mid S\right)\left(=\sup \left\{\left|\left(x^{* *}, x^{*}\right)-\left(x^{* *}, y^{*}\right)\right|: x^{*}, y^{*} \in S\right\}\right.$, the oscillation of $x^{* *}$ on $\left.S\right)<\varepsilon$.

If $K$ is weak ${ }^{*}-B\left(X^{* *}\right)$-dentable, then $K$ is said to be weak $k^{*}$ scalarly dentable.

Further, let us define a following notion as a generalization of $\delta$-Rademacher trees in $X^{*}$.

Definition 5. A system $\left\{x^{*}(n, k): n=0,1, \cdots ; k=1, \cdots, 2^{n}\right\}$ in $X^{*}$ is called a tree if $x^{*}(n, k)=\left\{x^{*}\left(n+1,2 k^{-1}\right)+x^{*}(n+1,2 k)\right\} / 2$ for $n=0,1, \cdots$, and $k=1, \cdots, 2^{n}$.

Let $A$ be a nonempty bounded subset of $X$. A tree $\left\{x^{*}(n, k): n=0,1, \cdots\right.$; $\left.k=1, \cdots, 2^{n}\right\}$ in $X^{*}$ is called an $A-\delta$-Rademacher tree if

$$
\sup _{x \in A}\left|\left(x, \sum_{k=1}^{2^{n-1}}(x *(n, 2 k-1)-x *(n, 2 k))\right)\right| \geqq 2^{n} \delta
$$


for every positive integer $n$.

If $A=B(X)$, then this tree is simply called a $\delta$-Rademacher tree.

In this paper, we are going to prove the following theorem which gives various characterizations of $K^{-}$weakly precompact sets $A$ by the effective use of the lifting theory $([7])$, weak ${ }^{*} \bar{A}^{*}$-dentability and a $K$-valued weak*measurable function constructed in the case where $A$ is a non- $K$-weakly precompact set. Viewing from our standpoint, we intend to give a direct and self-contained proof of Theorem as far as we can.

Theorem. Let $A$ be a bounded subset of $X$ and $K$ a weak ${ }^{*}$-compact subset of $X^{*}$. Then the following statements about $A$ and $K$ are equivalent.

(a) The set $A$ is $K$-weakly precompact.

(b) The set $\overline{c o} *(K)$ has the $\bar{A}^{*}-P C P$.

(c) For every weak*-measurable function $f: I \rightarrow K$ and every $E \in \Lambda^{+}$, the set $\overline{c o} *\left(T_{f}^{*}\left(\Delta_{E}\right)\right)$ is weak ${ }^{*}-\bar{A}^{*}$ - dentable.

(d) Every weak ${ }^{*}$-measurable function $f: I \rightarrow K$ is weak*-equivalent to a $\bar{A} *$ - measurable function $g: I \rightarrow \overline{c o} *(K)$.

( e ) For every bounded linear operator $T: C(K) \rightarrow L_{\infty}$, the set $\left(i^{\circ} T^{\circ} e\right)(A)$ is relatively norm compact (Here $i: L_{\infty} \rightarrow L_{1}$ is the canonical injection).

(f) For every weak $*_{-}$measurable function $f: I \rightarrow K$, the set $T_{f}(A)$ is relatively norm compact.

(g) For every weak $k^{*}$ measurable function $f: I \rightarrow K$, it holds that

$$
\inf _{n \geqq 1}\left\{\sup _{x \in A}\left|\left(x, T_{f}^{*}\left(r_{n}\right)\right)\right|\right\}=0
$$

(Here $r_{n}$ denotes the $n^{\text {th }}$ Rademacher function on $I$ ).

( $h$ ) For every weak $*^{*}$ measurable function $f: I \rightarrow K$, the set $T_{f}^{*}\left(\Delta_{I}\right)$ contains no $A$ - $\delta$-Rademacher tree.

The main point we wish to emphasize in Theorem is the equivalence among the statements (a), (c) and (d) (especially, implications: (c) $\Rightarrow(d)$ and (d) $\Rightarrow$ (a)). Our proof that (c) $\Rightarrow$ (d) is natural and meaningful, which is suggested by an approach due to Girardi and Uhl as stated above. The other implications can be regarded as generalizations of corresponding results already known when $K$ is a Pettis set (that is, $A=B(X)$ ). Further, in the case where $A$ is non- $K$-weakly precompact, we can prove that there exists a weak ${ }^{*}$-measurable function $h: I \rightarrow K$ that satisfies following all properties:

( $\alpha$ ) The function $h$ is not weak*-equivalent to any $\bar{A}^{*}$-measurable function $g: I \rightarrow X^{*}$. 
( $\beta) \quad \inf _{n \geqq 1}\left\{\sup _{x \in A}\left|\left(x, T_{h}^{*}\left(r_{n}\right)\right)\right|\right\}>0$.

( $\gamma$ ) The set $T_{h}{ }^{*}\left(\Delta_{I}\right)$ contains an $A$ - $\delta$-Rademacher tree for an appropriate positive number $\delta$.

$(\delta)$ The set $T_{h}(A)$ is not relatively norm compact.

( $\varepsilon$ ) For the bounded linear operator $T: C(K) \rightarrow L_{\infty}$ given by $T(f)=f \circ h$ for every $f \in C(K)$, the set $(i \circ T \circ e)(A)$ is not relatively norm compact.

In $\S 2$, we prepare some more notations and well-known facts that are necessary for the proof of Theorem. $\S 3$ is devoted to the proof of Theorem. In $\S 4$, as special cases of Theorem, various characterizations of Pettis sets, weak Radon-Nikodym sets and weakly precompact sets are presented (Of course, many of them have been already known. See, for instance, [22], [8], [9], [2], and [10]).

\section{§2. Preliminaries}

Let us recall the definition of the lifting on $L_{\infty}$. A lifting $\rho$ of $L_{\infty}$ is a map : $L_{\infty} \rightarrow M(I, \Lambda, \lambda)$ (the set of all bounded Lebesgue measurable functions on $I$ ) that is linear, multiplicative, positive such that $\rho(1)=1$, and such that $\rho(f)$ belongs to the class of $f$ for each $f \in L_{\infty}$. On $(I, \Lambda, \lambda)$, such liftings exist and so we always take an arbitrary, but fixed lifting $\rho$. For each $E \in \Lambda, \rho\left(\chi_{E}\right)$ is the characteristic function of a uniquely determined set belonging to $\Lambda$, which is denoted by $\rho(E)$. So we have $\chi_{\rho(E)}=\rho\left(\chi_{E}\right)$ for each $E \in \Lambda$. The map $\rho: \Lambda$ $\rightarrow \Lambda$ thus obtained satisfies that (1) $\rho(E) \equiv E$, (2) $\rho(E)=\rho(F)$ if $E \equiv F$, (3) $\rho(I)=I, \rho(\phi)=\phi$, (4) $\rho(E \cap F)=\rho(E) \cap \rho(F)$, and (5) $\rho(E \cup F)=$ $\rho(E) \cup \rho(F)$. Here $E \equiv F(E, F \in \Lambda)$ means that $\lambda((E \backslash F) \cup(F \backslash E))=0$.

Now we list two basic facts coming from the lifting theory. They are useful for the proof of Theorem. One is as follows. Let $K$ be a weak ${ }^{*}$-compact subset of $X^{*}$ and $f$ a weak*-measurable function from $I$ to $K$. Then, in virtue of the lifting theory, we have a weak*-measurable function $\theta(f): I \rightarrow \overline{c o} *(K)$ such that $(x, \theta(f)(t))=\rho(x \circ f)(t)$ for every $x \in X$ and every $t \in I$. Note that $\sup _{t \in E}(x, \theta(f)(t))=$ ess $^{-} \sup _{t \in E}(x, f(t))$ for every $E \in \Lambda^{+}$with $\rho(E)=E$. The other is a following fact, which we state as Proposition 1. Let $Y$ be a compact Hausdorff space and let $k: I \rightarrow Y$ be a function such that $f \circ k$ is $\lambda$-measurable for every $f \in C(Y)$. Then, in virtue of the lifting theory, we have:

Proposition 1. If $\rho(f \circ k)=f \circ k$ for every $f \in C(Y)$, then $k$ is $\Lambda-\mathscr{B}(Y)$ ( : the Borel $\sigma$-algebra of $Y$ ) measurable and $k(\lambda)$ (: the image measure of $\lambda$ by $k$ ) is a Radon measure on $Y$. 
Next let us note two basic facts on the measurability of functions, which we state as Proposition 2 and Proposition 3. Proposition 3 can be proved easily by the exhaustion.

Proposition 2. Let $Y$ and $Z$ be compact Hausdorff spaces, $\alpha$ a Borel measure on $Y$ and $f: Y \rightarrow Z$ a $\mathscr{B}(Y)-\mathscr{B}(Z)$ measurable function. If $g: Z \rightarrow R$ (the set of real numbers) is $f(\alpha)$-measurable, then $g \circ f$ is $\alpha$-measurable.

Proposition 3. Let $f: I \rightarrow R$ be a function. Then $f$ is $\lambda$-measurable if and only if $f$ satisfies the following property $(*)$.

(*) For every $\varepsilon>0$ and every $E \in \Lambda^{+}$, there exists an element $F$ of $\Lambda^{+}$such that $F \subset E$ and $0(f \mid F)<\varepsilon$.

\section{\$3. Proof of Theorem}

We are going to prove that (a) $\Rightarrow(\mathrm{b}) \Rightarrow(\mathrm{c}) \Rightarrow(\mathrm{d}) \Rightarrow(\mathrm{a})$ and (a) $\Rightarrow$ (e) $\Rightarrow(\mathrm{f}) \Rightarrow(\mathrm{g}) \Rightarrow(\mathrm{a}) \Rightarrow$ (h) $\Rightarrow$ (a).

(i) We begin with proving that there exists a weak*-measurable function $h: I \rightarrow K$ that satisfies the following properties $(\alpha),(\beta)$ and $(\gamma)$, if $A$ is not $K$-weakly precompact.

( $\alpha$ ) The function $h$ is not weak*-equivalent to any $\bar{A}^{*}$-measurable function $g: I \rightarrow X^{*}$.

( $\beta) \quad \inf _{n \geqq 1}\left\{\sup _{x \in A}\left|\left(x, T_{h}^{*}\left(r_{n}\right)\right)\right|\right\}>0$.

( $\gamma$ ) The set $T_{h}{ }^{*}\left(\Delta_{I}\right)$ contains an $A$ - $\delta$-Rademacher tree for an appropriate positive number $\delta$.

Then we know that $(\mathrm{d}) \Rightarrow(\mathrm{a}),(\mathrm{g}) \Rightarrow(\mathrm{a})$, and $(\mathrm{h}) \Rightarrow(\mathrm{a})$.

Now let us construct this function $h$ by the same way as in [10]. For the sake of completeness and necessity, let us state its construction in brief. Since $A$ is not $K$-weakly precompact, there exists a sequence $\left\{x_{n}\right\}_{n \geq 1}$ in $A$ having no pointwise convergent subsequence on $K$. Therefore, by the celebrated argument of Rosenthal [17], we have a subsequence $\left\{x_{n(m)}\right\}_{m \geq 1}$ of $\left\{x_{n}\right\}_{n \geq 1}$ and real numbers $r$ and $\delta$ with $\delta>0$ such that putting $A_{m}=\left\{x^{*} \in K:\left(x^{*}, x_{n(m)}\right) \leqq r\right\}$ and $B_{m}=\left\{x^{*} \in K:\left(x^{*}, x_{n(m)}\right) \geqq r+\delta\right\}$, then $\left(A_{m}, B_{m}\right)_{m \geq 1}$ is an independent sequence of pairs of closed subsets of $K$ (that is, for every $\left\{\varepsilon_{j}\right\}_{1 \leq j \leq k}$ with $\varepsilon_{j}=1$ or $-1, \cap\left\{\varepsilon_{j} A_{j}: 1 \leqq j \leqq k\right\}$ is a nonempty set, where $\varepsilon_{j} A_{j}=A_{j} \quad$ if $\varepsilon_{j}=1$ and $\varepsilon_{j} A_{j}=B_{j} \quad$ if $\left.\quad \varepsilon_{j}=-1\right)$. Put $\Gamma=\bigcap_{m \geq 1}\left(A_{m} \cup B_{m}\right)$. Then $\Gamma$ is a nonempty compact subset of $K$, since $\left(A_{m}, B_{m}\right)_{m \geq 1}$ is independent. Define $\phi: \Gamma \rightarrow \mathscr{P}(N)$ 
(Cantor space, with its usual compact metric topology) by $\phi\left(x^{*}\right)=\left\{p:\left(x_{n}(p), x^{*}\right)\right.$ $\leqq r\}\left(=\left\{p: A_{p} \ni x^{*}\right\}\right) \in \mathscr{P}(N)$. Then $\phi$ is a continuous surjection and so we have a Radon probability measure $\gamma$ on $\Gamma$ such that $\phi(\gamma)=\nu$ (the normalized Haar measure if we identify $\mathscr{P}(N)$ with $\left.\{0,1\}^{N}\right)$ and $\left\{f \circ \phi: f \in L_{1}(\mathscr{P}(N)\right.$, $\left.\left.\sum_{\nu}, \nu\right)\right\}=L_{1}\left(\Gamma, \Sigma_{\gamma}, \gamma\right)$ where $\sum_{\nu}\left(\right.$ resp. $\left.\sum_{\gamma}\right)$ is the family of all $\nu$ (resp. $\gamma$ ) measurable subsets of $\mathscr{P}(N)$ (resp. $\Gamma$ ). Further, consider a function $\tau: \mathscr{P}(N)$ $\rightarrow I$ defined by $\tau(B)=\sum\left\{1 / 2^{m}: m \in B\right\}$ for every $B \in \mathscr{P}(N)$. Then $\tau$ is a continuous surjection such that $\tau(\nu)=\lambda$ and $\left\{u \circ \tau: u \in L_{1}(I, \Lambda, \lambda)\right\}=$ $L_{1}\left(\mathscr{P}(N), \sum_{\nu}, \nu\right)$. Define a linear operator $S: L_{1} \rightarrow L_{1}\left(\Gamma, \Sigma_{\gamma}, \gamma\right)$ by $S(u)=u \circ \tau$ 。 $\phi$ for every $u \in L_{1}$. Then the operator $S$ is a surjective isometry such that $S^{*}(g)\left(\tau\left(\phi\left(x^{*}\right)\right)\right)=g\left(x^{*}\right) \quad \gamma$-a.e. on $\Gamma$ for every $g \in L_{\infty}\left(\Gamma, \Sigma_{\gamma}, \gamma\right)$ and $S^{*}\left(g_{1} \cdot g_{2}\right)=\mathrm{S}^{*}\left(g_{1}\right) \cdot S^{*}\left(g_{2}\right)$ in $L_{\infty}$ for $g_{1}, g_{2} \in L_{\infty}\left(\Gamma, \sum_{\gamma}, \gamma\right)$. Let $\rho$ be a lifting of $L_{\infty}$. For each $t \in I$, the bounded linear functional $L_{t}$ on $C(\Gamma)$ defined by $L_{t}(f)=\rho\left(S^{*}(f)\right)(t)$ for every $f \in C(\Gamma)$ is multiplicative, and so we have a function $h: I \rightarrow \Gamma(\subset K)$ such that $f(h(t))=\rho\left(S^{*}(f)\right)(t)$ for every $f \in C(\Gamma)$ and every $t \in I$. Hence we easily know that $h$ is weak*-measurable. Now let us show that this function $h$ has the above properties $(\alpha),(\beta)$ and $(\gamma)$.

In order to prove the property $(\alpha)$, we first note that

$$
\rho(f \circ h)(t)=\rho(S *(f))(t)=(f \circ h)(t)
$$

for every $f \in C(\Gamma)$ and every $t \in I$. Then, by Proposition $1, h$ is a $\Lambda-\mathscr{B}(\Gamma)$ measurable function and $h(\lambda)$ is a Radon measure on $\Gamma$. So we have that $h(\lambda)$ $=\gamma$. Further, since $S^{*}(f)(t)=(f \circ h)(t) \lambda$-a.e. on $I$ for every $f \in C(\Gamma)$, we have that

$$
(f \circ h \circ \tau \circ \phi)\left(x^{*}\right)=S^{*}(f)\left(\tau\left(\phi\left(x^{*}\right)\right)\right)=f\left(x^{*}\right) \quad r \text {-a.e. on } \Gamma
$$

for every $f \in C(\Gamma)$. Hence, for every $m$ there exists an $E_{m} \in \mathscr{B}(\Gamma)$ such that $\gamma\left(E_{m}\right)=0$ and $\left(x_{n(m)},(h \circ \tau \circ \phi)\left(x^{*}\right)\right)=\left(x_{n(m)}, x^{*}\right)$ for every $x^{*} \in E_{m}$, where $\left\{x_{n(m)}\right\}_{m \geq 1}$ is a sequence in $A$ obtained above (as a subsequence of $\left\{x_{n}\right\}_{n \geq 1}$ ). Therefore, we have an $E \in \mathscr{B}(\Gamma)$ such that $\gamma(E)=0$ and $\left(x_{n}(m),(h \circ \tau \circ \phi)\left(x^{*}\right)\right)$ $=\left(x_{n(m)}, x^{*}\right)$ for every $x^{*} \in E$ and every $m$. So, letting $x^{* *}\left(\in \bar{A}^{*}\right)$ be a weak*-cluster point of $\left\{x_{n(m)}\right\}_{m \geq 1}$, we have that

$$
\left(x^{* *},(h \circ \tau \circ \phi)\left(x^{*}\right)\right)=\left(x^{* *}, x *\right) \gamma \text {-a.e. on } \Gamma
$$

Take such a point $x^{* *}$ so that $x^{* *} \mid \Gamma$ is expressible as $\lim _{m \rightarrow \mathscr{F}} x_{n(m)} \mid \Gamma$ for some non-principal ultrafilter $\mathscr{F}$ on $N$. Then we see that for $x^{*} \in \Gamma$

$$
\left(x^{* *}, x^{*}\right) \leqq r \Leftrightarrow\left\{m:\left(x_{n(m)}, x^{*}\right) \leqq r\right\} \in \mathscr{F} \Leftrightarrow \phi\left(x^{*}\right) \in \mathscr{F}
$$


which implies that $\left\{x^{*}:\left(x^{* *}, x^{*}\right) \leqq \gamma\right\}=\phi^{-1}(\mathscr{F})$ and so $x^{* *}$ is not $\gamma$-measurable, since $\mathscr{F}$ is not $\nu$-measurable and $\phi(\gamma)=\nu$. Hence we get that $x^{* *} \circ h \circ \tau \circ \phi$ is not $\gamma$-measurable. Thus, by virtue of Proposition $2, x * * \circ h$ is not $\tau(\phi(\gamma))(=\lambda)$-measurable.

Suppose that there exists a $\bar{A}^{*}$-measurable function $g: I \rightarrow \overline{c o}^{*}(K)$ such that $h-g$ is weak*-scalarly null. Then we easily get that $x^{* * \circ} h=x^{* * \circ g}$ $\lambda$-a.e. on $I$. This contradicts the fact that $x^{* * \circ g}$ is $\lambda$-measurable. So we complete the proof of the property $(\alpha)$.

In order to prove the property $(\beta)$, we first note that

$$
\begin{aligned}
& \int_{E} f(h(t)) d \lambda(t)=\int_{E} \rho\left(S^{*}(f)\right)(t) d \lambda(t)=\int_{E} S^{*}(f)(t) d \lambda(t) \\
& =\int_{E} S^{*}(f)(t) d(\tau(\phi(\gamma)))(t) \\
& =\int_{\phi^{-1}\left(\tau^{-1}(E)\right)} S^{*}(f)\left(\tau\left(\phi\left(x^{*}\right)\right)\right) d \gamma\left(x^{*}\right) \\
& =\int_{\phi^{-1}\left(\tau^{-1}(E)\right)} f\left(x^{*}\right) d \gamma\left(x^{*}\right)
\end{aligned}
$$

for every $E \in \Lambda$ and every $f \in C(\Gamma)$.

Let $\left\{I(m, k): m=0,1, \cdots ; k=1, \cdots, 2^{m}\right\}$ be a system of intervals in $I$ given by $I(m, k)=\left[(k-1) / 2^{m}, k / 2^{m}\right)$ if $m \geqq 1,1 \leqq k \leqq 2^{m}-1$ and $I\left(m, 2^{m}\right)$ $=\left[\left(2^{m}-1\right) / 2^{m}, 1\right]$ if $m \geqq 0$. Then we get that $\phi^{-1}\left(\tau^{-1}(I(m, 2 k-1))\right) \subset B_{m}$ and $\phi^{-1}\left(\tau^{-1}(I(\mathrm{~m}, 2 k))\right) \subset A_{m}$ for $m=1,2, \cdots$ and $k=1, \cdots, 2^{m-1}$. Take the sequence $\left\{x_{n(m)}\right\}_{m \geq 1}$ in $A$ obtained above. Then, since

$$
\gamma_{m}(t)=\sum_{k=1}^{2^{m}}(-1)^{k-1} \chi_{I(m, k)}(t),
$$

we have that for every $m$

$$
\begin{aligned}
& \left(x_{n(m)}, T_{h} *\left(r_{m}\right)\right)=\left(T_{h}\left(x_{n(m)}\right), r_{m}\right) \\
& =\int_{I}\left(x_{n(m)}, h(t)\right) \cdot r_{m}(t) d \lambda(t) \\
& =\sum_{k=1}^{2^{m}}(-1)^{k-1} \cdot \int_{I(m, k)}\left(x_{n(m)}, h(t)\right) d \lambda(t)
\end{aligned}
$$




$$
\begin{aligned}
& =\sum_{k=1}^{2^{m-1}} \int_{I(m, 2 k-1)}\left(x_{n(m)}, h(t)\right) d \lambda(t) \\
& -\sum_{k=1}^{2^{m-1}} \int_{I(m, 2 k)}\left(x_{n}(m), h(t)\right) d \lambda(t) \\
& =\sum_{k=1}^{2^{m-1}}\left\{\int_{\phi^{-1}\left(\tau^{-1}(I(m, 2 k-1))\right)}\left(x_{\left.n(m), x^{*}\right) d \gamma\left(x^{*}\right)}-\int_{\phi^{-1}\left(\tau^{-1}(I(m, 2 k))\right)}\left(x_{n}(m), x^{*}\right) d \gamma\left(x^{*}\right)\right\}\right. \\
& \geqq \sum_{k=1}^{2^{m-1}}\left\{(r+\delta)\left(1 / 2^{m}\right)-r\left(1 / 2^{m}\right)\right\}=\delta / 2 .
\end{aligned}
$$

Hence we have that

$$
\inf _{m \geqq 1}\left\{\sup _{x \in A}\left|\left(x, T_{h}^{*}\left(r_{m}\right)\right)\right|\right\} \geqq \delta / 2 \text {, }
$$

which is the desired result.

In order to prove the property $(\gamma)$, let $x^{*}(m, k)=T_{h} *\left(\chi_{I(m, k)} / \lambda(I(m, k))\right)$ $=2^{m} \cdot T_{h}^{*}\left(\chi_{I(m, k)}\right)$ for $m=0,1, \cdots$ and $k=1, \cdots, 2^{m}$. Then we know that $\left\{x *(m, k): m=0,1, \cdots ; k=1, \cdots, 2^{m}\right\}$ is a tree in $X^{*}$. Indeed, it holds that $x *(m, k)=\{x *(m+1,2 k-1)+x *(m+1,2 k)\} / 2$ for $m=0,1, \cdots$ and $k=1$, $\cdots, 2^{m}$. Now, take the sequence $\left\{x_{n(m)}\right\}_{m \geq 1}$ in $A$ obtained above. Then we have that for $\mathrm{m}=1,2, \cdots$

$$
\begin{aligned}
& \sup _{x \in A}\left|\left(x, \sum_{k=1}^{2^{m-1}}(x *(m, 2 k-1)-x *(m, 2 k))\right)\right| \\
& \geqq\left|\left(x_{n}(m), \sum_{k=1}^{2^{m-1}}\left(x^{*}(m, 2 k-1)-x *(m, 2 k)\right)\right)\right| \\
& =2^{m} \cdot\left|\left(x_{n(m)}, \sum_{k=1}^{2^{m-1}}\left(T_{h}^{*}\left(\chi_{I(m, 2 k-1)}\right)-T_{h} *\left(\chi_{I(m, 2 k)}\right)\right)\right)\right| \\
& \geqq 2^{m} \cdot\left(x_{n(m)}, \sum_{k=1}^{2^{m-1}}\left(T_{h}^{*}\left(\chi_{I(m, 2 k-1)}\right)-T_{h}^{*}\left(\chi_{I(m, 2 k)}\right)\right)\right) \\
& =2^{m} \cdot\left\{\sum_{k=1}^{2^{m-1}} \int_{I(m, 2 k-1)}\left(x_{n}(m), h(t)\right) d \lambda(t)\right.
\end{aligned}
$$




$$
\begin{aligned}
& \left.-\sum_{k=1}^{2^{m-1}} \int_{I(m, 2 k)}\left(x_{n(m)}, h(t)\right) d \lambda(t)\right\} \\
& =2^{m} \cdot\left(x_{n(m)}, T_{h} *\left(r_{m}\right)\right) \geqq 2^{m}(\delta / 2) .
\end{aligned}
$$

Hence, putting $\eta=\delta / 2$, the set $T_{h} *\left(\Delta_{I}\right)$ contains an $A-\eta$-Rademacher tree, and so the proof is completed.

(ii) (a) $\Rightarrow$ (b). Assume that (a) is true. Then it easily follows that $A$ is $\overline{c o}^{*}(K)$-weakly precompact. Suppose that there exists an element $x^{* *}$ of $\bar{A}^{*}$ and a weak*-compact subset $M$ of $\overline{c o}{ }^{*}(K)$ such that the restriction of $x * *$ to $M$ has no point of continuity. Then, in virtue of Lemmas 2 and 3 in [15], we know that there exists a nonempty compact subset $L$ of $M$, real numbers $r$ and $\delta$ with $\delta>0$ and a sequence $\left\{x_{n}\right\}_{n \geq 1}$ in $A$ such that putting $A_{n}=\left\{x * \in L:\left(x_{n}\right.\right.$, $\left.\left.x^{*}\right) \leqq r\right\}$ and $B_{n}=\left\{x^{*} \in L:\left(x_{n}, x^{*}\right) \geqq r+\delta\right\}$, then $\left(A_{n}, B_{n}\right)_{n \geq 1}$ is independent. This implies that a sequence $\left\{x_{n}\right\}_{n \geq 1}$ in $A$ has no pointwise convergent subsequence on $M$. This contradicts the fact that $A$ is $\overline{c o}^{*}(K)$-weakly precompact. Thus we get that $(\mathrm{a}) \Rightarrow(\mathrm{b})$.

(iii) (b) $\Rightarrow$ (c). Suppose that (c) fails. Then there exist a weak ${ }^{*}$-measurable function $f: I \rightarrow K$ and an element $E$ of $\Lambda^{+}$such that $M\left(=\overline{c o}^{*}\left(T_{f}^{*}\left(\Delta_{E}\right)\right)\right)$ $\left.\subset \overline{c o}^{*}(K)\right)$ is not weak ${ }^{*}-\bar{A}^{*}$-dentable. Hence there exists a positive number $\varepsilon$ and an element $x^{* *}$ of $\bar{A}^{*}$ such that $0\left(x^{* *} \mid S\right) \geqq \varepsilon$ for every weak ${ }^{*}$-open slice $S$ of $M$. Then it holds that $0\left(x^{* *} \mid V \cap M\right) \geqq \varepsilon$ for every weak*-open subset $V$ of $X^{*}$ with $V \cap M \neq \phi$. Indeed, take such a set $V$. By Lemma II. 1 in [5], there exist positive numbers $\alpha_{1}, \cdots, \alpha_{p}$ whose sum is one and weak*-open slices $S_{1}, \cdots, S_{p}$ of $M$ such that

$$
\sum_{i=1}^{p} \alpha_{i} S_{i} \subset V \cap M
$$

So we have that

$$
\varepsilon \leqq \sum_{i=1}^{p} \alpha_{i} 0\left(x * * \mid S_{\imath}\right)=0\left(x^{* * \mid} \sum_{i=1}^{p} \alpha_{i} S_{i}\right) \leqq 0(x * * \mid V \cap M)
$$

for every weak*-open subset $V$ of $X^{*}$ with $V \cap M \neq \phi$. Hence the restriction of $x^{* *}$ to $M$ has no point of continuity. So (b) fails. Thus we see that (b) $\Rightarrow$ (c).

(iv) (c) $\Rightarrow(d)$. Assume that (c) holds. Take a weak ${ }^{*}$-measurable function $f: I \rightarrow K$. Then, by the remark stated in $\S 2$, we have a weak ${ }^{*}$-measurable 
function $\theta(f): I \rightarrow \overline{c o}^{*}(K)$ such that $\rho(x \circ f)=x \circ \theta(f)$ for every $x \in X$. Hence $f$ is weak*-equivalent to $\theta(f)$. So let us show that $x^{* * \circ} \theta(f)$ is $\lambda$-measurable for every $x^{* *} \in \bar{A}^{*}$. In virtue of Proposition 3, we have only to show that for every $\varepsilon>0$ and every $E \in \Lambda^{+}$, there is a subset $F$ of $E$ such that $F \in \Lambda^{+}$and $0\left(x^{* * \circ} \theta(f) \mid F\right)<\varepsilon$. Fix $x^{* *} \in \bar{A}^{*}, \varepsilon>0$ and $E \in \Lambda^{+}$, and set $D=\rho(E)\left(\in \Lambda^{+}\right)$. Since $M\left(=\overline{c o}^{*}\left(T_{f}^{*}\left(\Delta_{D}\right)\right)\right)$ is weak ${ }^{*}-\bar{A}^{*}$-dentable by the assumption, there exists a weak*-open slice $S(=S(x, \alpha, M))$ of $M$ such that $0\left(x^{* *} \mid S\right)<\varepsilon$. Then we have that

$$
\begin{aligned}
S= & S(x, \alpha, M) \\
= & \left\{x^{*} \in M:\left(x, x^{*}\right)>\sup _{y^{\prime} \in M}\left(x, y^{*}\right)-\alpha\right\} \\
= & \left\{x^{*} \in M:\left(x, x^{*}\right)>\sup _{y^{*} \in T_{f}^{*}\left(\Delta_{D}\right)}\left(x, y^{*}\right)-\alpha\right\} \\
= & \left\{x^{*} \in M:\left(x, x^{*}\right)>\right. \\
& \left.\sup \left(\int_{G}(x, f(t)) d \lambda(t) / \lambda(G): G \subset D, G \in \Lambda^{+}\right)-\alpha\right\} \\
= & \left\{x^{*} \in M:\left(x, x^{*}\right)>\operatorname{ess}^{-} \sup (x, f(t))-\alpha\right\} \\
= & \left\{x^{*} \in M:\left(x, x^{*}\right)>\sup _{t \in D}(x, \theta(f)(t))-\alpha\right\},
\end{aligned}
$$

as $\rho(D)=D . \quad$ Set $F_{0}=\left\{s \in D:(x, \theta(f)(s))>\sup _{t \in D}(x, \theta(f)(t))-\alpha\right\}$. Then $F_{0} \in \Lambda^{+}$, since $\sup _{t \in D}(x, \theta(f)(t))=$ ess- $\sup _{t \in D}(x, \theta(f)(t))$. Furthermore, we have that $\theta(f)\left(F_{0}\right) \subset M$. Indeed, suppose that there exists an element $s$ of $F_{0}$ such that $\theta(f)(s) \in M$. Then, by the separation theorem, there exists an element $a$ of $X$ such that

$$
(a, \theta(f)(s))>\beta=\sup _{x^{\prime} \in M}\left(a, x^{*}\right) .
$$

That is, we have that

$$
\begin{aligned}
(a, \theta(f)(s))>\beta & =\sup \left\{\left(a, T_{f}^{*}\left(\chi_{G}\right) / \lambda(G)\right): G \subset D, G \in \Lambda^{+}\right\} \\
& =\operatorname{ess}^{-} \sup _{t \in D}(a, f(t)) \\
& =\sup _{t \in D}(a, \theta(f)(t)),
\end{aligned}
$$

which is a contradiction. Thus we know that $\theta(f)\left(F_{0}\right) \subset S$ and so, $0\left(x^{* * \circ} \theta(f) \mid F_{0}\right)$ $=0\left(x * * \mid \theta(f)\left(F_{0}\right)\right) \leqq 0(x * * \mid S)<\varepsilon$. Finally, letting $F=F_{0} \cap E$, we easily get that $F$ is a desired set, since $D=\rho(E) \equiv E$. This completes the proof.

(v) (a) $\Rightarrow$ (e). In order to prove (e), take an arbitrary sequence $\left\{x_{n}\right\}_{n \geq 1}$ in $A$ and set $U=T \circ e\left(: X \rightarrow L_{\infty}\right)$. Then there exists a weak ${ }^{*}$-measurable 
function $f: I \rightarrow \overline{c o}^{*}(K)$ such that

$$
\left(x, U^{*}\left(\chi_{E}\right)\right)=\int_{E}(x, f(t)) d \lambda(t)
$$

for every $E \in \Lambda$ and every $x \in X$. Hence it follows that $U(x)(t)=(x, f(t))$ $\lambda$-a.e. on $I$ for every $x \in X$, and so, there exists a null set $E$ satisfying $U\left(x_{n}\right)(t)$

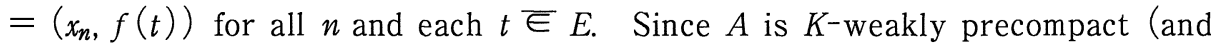
so, $A$ is $\overline{c o}^{*}(K)$-weakly precompact), there exists a pointwise convergent subsequence $\left\{x_{n(k)}\right\}_{k \geq 1}$ on $\overline{c o}^{*}(K)$. Hence $U\left(x_{n(k)}\right)$ converges $\lambda$-a.e. on $I$ and by boundedness $i \circ T \circ e\left(x_{n(k)}\right)\left(=i \circ U\left(x_{n(k)}\right)\right)$ must converge in $L_{1}$. Thus the set $(i \circ T \circ e)(A)$ is relatively norm compact.

(vi) (e) $\Rightarrow(\mathrm{f})$. Suppose that (e) is true. Take a weak*-measurable function $f: I \rightarrow K$ and define a bounded linear operator $T: C(K) \rightarrow L_{\infty}$ by $T(g)$ $=g \circ f$ for every $g \in C(K)$. Then we get that $T(e(x))=e(x) \circ f=x \circ f$ for every $x \in X$. Hence we see that $T_{f}(A)=(i \circ T \circ e)(\mathrm{A})$ and so, the set $T_{f}(A)$ is relatively norm compact by the assumption.

(vii) $(\mathrm{f}) \Rightarrow(\mathrm{g})$. Assume that $(f)$ is true. Take a weak ${ }^{*}$-measurable function $f: I \rightarrow K$ and let $\varepsilon>0$. Since the set $T_{f}(A)$ is relatively norm compact, there exists $x_{1}, \cdots, x_{m} \in A$ such that

$$
T_{f}(A) \subset \bigcup_{i=1}^{m}\left\{g:\left\|g-T_{f}\left(x_{i}\right)\right\|<\varepsilon / 2\right\} .
$$

Since $\lim _{n \rightarrow \infty} \int_{I}\left(x_{i}, f(t)\right) \cdot v_{n}(t) d \lambda(t)=0$ for every $i$ with $1 \leqq i \leqq m$, we can choose an integer $p$ such that if $n>p$ then

$$
\left|\int_{I}\left(x_{i}, f(t)\right) \cdot r_{n}(t) d \lambda(t)\right|<\varepsilon / 2
$$

for every $i$ with $1 \leqq i \leqq m$. Let $x \in A$ and let $x_{i}$ be such that $\left\|T_{f}(x)-T_{f}\left(x_{i}\right)\right\|$ $<\varepsilon / 2$. Then, if $n>p$, we have that

$$
\begin{aligned}
& \left|\left(x, T_{f}^{*}\left(r_{n}\right)\right)\right|=\left|\left(T_{f}(x), r_{n}\right)\right| \\
& =\left|\int_{I}(x, f(t)) \cdot v_{n}(t) d \lambda(t)\right| \\
& =\left|\int_{I}\left\{(x, f(t))-\left(x_{i}, f(t)\right)\right\} \cdot v_{n}(t) d \lambda(t)+\int_{I}\left(x_{i}, f(t)\right) \cdot r_{n}(t) d \lambda(t)\right| \\
& \leqq \int_{I}\left|(x, f(t))-\left(x_{i}, f(t)\right)\right| d \lambda(t)+\left|\int_{I}\left(x_{i}, f(t)\right) \cdot v_{n}(t) d \lambda(t)\right| \\
& =\| T_{f}(x)-T_{f}\left(x_{i}\right)||+\left|\int_{I}\left(x_{i}, f(t)\right) \cdot r_{n}(t) d \lambda(t)\right| \\
& <\varepsilon / 2+\varepsilon / 2=\varepsilon .
\end{aligned}
$$


Hence we see that $(\mathrm{g})$ is true.

(viii) (a) $\Rightarrow(\mathrm{h})$. Suppose that (a) is true. Then $A$ is $\overline{c o}^{*}(K)$-weakly precompact. Hence, by the equivalence between (a) and (g) already proved, we have that $\inf \left\{\sup _{n \geq 1}\left|\left(x, T_{f}^{*}\left(r_{n}\right)\right)\right|\right\}=0$ for every weak*-measurable function $f: I \rightarrow \overline{c o}^{*}(K)^{n}$. Suppose that $(\mathrm{h})$ fails. Then we have a weak ${ }^{*}$-measurable function $u: I \rightarrow K$ such that $T_{u}^{*}\left(\Delta_{I}\right)$ contains an $A$ - $\delta$-Rademacher tree $\left\{x^{*}(n, k)\right.$ : $\left.n=0,1, \cdots ; k=1, \cdots, 2^{n}\right\}$. Note that this tree is contained in $\overline{c o}^{*}(K)$ by the separation theorem. Let $\left(g_{n}, \Lambda_{n}\right)_{n \geq 1}\left(\Lambda_{n}\right.$ : the $\sigma$-algebra generated by $\{I(n, k): k$ $\left.=1, \cdots, 2^{n}\right\}$ ) be the dyadic martingale associated with this $A$ - $\delta$-Rademacher tree $\left\{x^{*}(n, k): n=0,1, \cdots ; k=1, \cdots, 2^{n}\right\}$. That is, each $g_{n}$ has the form

$$
g_{n}=\sum_{k=1}^{2^{n}} x^{*}(n, k) \chi_{I(n, k)}
$$

Then we can define a measure $\alpha: \Lambda \rightarrow X^{*}$ satisfying that $\alpha(E)=$ $\lim _{n \rightarrow \infty} \int_{E} g_{n}(t) d \lambda(t)$ for every $E \in \Lambda$. Since $\alpha(E) \in \lambda(E) \cdot \overline{c o}^{*}(K)$ for every $E \in \Lambda$, there exists a weak ${ }^{*}$-measurable function $f: I \rightarrow \overline{c o}^{*}(K)$ such that

$$
(x, \alpha(E))=\int_{E}(x, f(t)) d \lambda(t)
$$

for every $x \in X$ and every $E \in \Lambda$. Then we have that for every $x \in X$

$$
\begin{aligned}
& \left(x, T_{f}^{*}\left(r_{n}\right)\right) \\
& =\int_{I}(x, f(t)) \cdot v_{n}(t) d \lambda(t) \\
& =\sum_{k=1}^{2^{n-1}}\left\{\int_{I(n, 2 k-1)}(x, f(t)) d \lambda(t)-\int_{I(n, 2 k)}(x, f(t)) d \lambda(t)\right\} \\
& =\sum_{k=1}^{2^{n-1}}\left\{\int_{I(n, 2 k-1)}\left(x, g_{n}(t)\right) d \lambda(t)-\int_{I(n, 2 k)}\left(x, g_{n}(t)\right) d \lambda(t)\right\}
\end{aligned}
$$

since $E\left(x \circ f \mid \Lambda_{n}\right)=x \circ g_{n}$ for every $x \in X$. Hence we have that for every $n$

$$
\begin{aligned}
& \sup _{x \in A}\left|\left(x, T_{f}^{*}\left(r_{n}\right)\right)\right| \\
& =\sup _{x \in A}\left|\sum_{k=1}^{2^{n-1}}\left(1 / 2^{n}\right) \cdot\left\{\left(x, x^{*}(n, 2 k-1)\right)-(x, x *(n, 2 k))\right\}\right|
\end{aligned}
$$




$$
\begin{aligned}
& =\left(1 / 2^{n}\right) \sup _{x \in A}\left|\left(x, \sum_{k=1}^{2^{n-1}}\{x *(n, 2 k-1)-x *(n, 2 k)\}\right)\right| \\
& \geqq\left(1 / 2^{n}\right)\left(2^{n} \delta\right)=\delta .
\end{aligned}
$$

This is a contradiction. So the proof is completed.

Consequently, we have completed all proofs of Theorem.

Remark 1. Let $h$ be the function constructed above. Combining the property $(\beta)$ in (i ) with the result (vii), we know that the set $T_{h}(A)$ is not relatively norm compact. But this fact can be proved more directly as follows (Note that this argument is the same as in the proof of Proposition (14-1-3) in [22]). Let $\left\{x_{n(m)}\right\}_{m \geq 1}$ be the sequence in $A$ obtained above. Then it holds that for $p<q$

$$
\begin{aligned}
& \| T_{h}\left(x_{n}(p)\right)-T_{h}\left(x_{n(q)}\right)|| \\
& =\int_{I}\left|\left(x_{n(p)}-x_{n(q)}, h(t)\right)\right| d \lambda(t) \\
& =\int_{\phi^{-1}\left(\tau^{-1}(I)\right)}\left|\left(x_{n(p)}-x_{n(q)}, x^{*}\right)\right| d \gamma\left(x^{*}\right) \\
& =\int_{\Gamma}\left|\left(x_{n(p)}-x_{n(q)}, x^{*}\right)\right| d \gamma\left(x^{*}\right) \\
& \geqq \int_{\Gamma \cap A_{p} \cap B_{q}}\left|\left(x_{n(p)}-x_{n(q),} x^{*}\right)\right| d \gamma\left(x^{*}\right) \\
& \quad+\int_{\Gamma \cap A_{q} \cap B_{p}}\left|\left(x_{n}(p)-x_{n(q)}, x^{*}\right)\right| d \gamma\left(x^{*}\right) \\
& =\int_{\Gamma \cap A_{p} \cap B_{q}}\left(x_{n(q)}-x_{n(p)}, x^{*}\right) d \gamma\left(x^{*}\right) \\
& \geqq \delta \cdot \gamma\left(\Gamma \cap A_{p} \cap B_{q}\right)+\delta \cdot \gamma\left(\Gamma \cap A_{q} \cap B_{p}\right) \\
& =\delta / 4+\delta / 4=\delta / 2,
\end{aligned}
$$

which implies that the set $T_{h}(A)$ is not relatively norm compact.

Furthermore, combining the proof of (vi) with this result, we get that for the bounded linear operator $T: C(K) \rightarrow L_{\infty}$ defined by $T(f)=f \circ h$ for every $f$ $\in C(K)$, the set $(i \circ T \circ e)(A)$ is not relatively norm compact.

Finally, the fact that $h$ is not weak*-equivalent to any $\bar{A}^{*}$-measurable function also can be proved as follows (That is, it is a proof depending on the non-relative norm compactness of the set $\left.T_{h}(A)\right)$. Suppose that there exists a $\bar{A}^{*}$-measurable function $g: I \rightarrow \overline{c o}^{*}(K)$ such that $h-g$ is weak*-scalarly null. Then it holds that $T_{h}(x)=T_{g}(x)$ for every $x \in X$. Well, the set $T_{g}(A)$ is relatively norm compact in virtue of a deep result due to Fremlin (Theorem $2 \mathrm{~F}$ in 
[4]), since $g$ is $\bar{A}^{*}$-measurable. Thus we get that the set $T_{h}(A)$ is relatively norm compact, which is a contradiction. Hence we see that (d) $\Rightarrow$ (a) in Theorem. Compared with this proof, our proof that (d) $\Rightarrow$ (a) in Theorem given before is more direct and easier, since it goes without invoking any deep result and thought, only depending on the definition of $\bar{A}^{*}$-measurability.

\section{§4. Pettis Sets, Weak Radon-Nikodym Sets and Weak Precompactness}

We collect results obtained as corollaries of Theorem. Setting $A=B(X)$ in Theorem, we immediately get various characterizations of Pettis sets as follows.

Corollary 1. Let $K$ be a weak*-compact subset of $X^{*}$. Then the following statements about $K$ are equivalent.

(a) The set $K$ is a Pettis set.

(b) The set $\overline{c o}^{*}(K)$ has the scalar point of continuity property.

(c) For every weak-measurable function $f: I \rightarrow K$ and every $E \in \Lambda^{+}$, the set $\overline{c o}^{*}\left(T_{f}^{*}\left(\Delta_{E}\right)\right)$ is weak ${ }^{*}$ scalarly dentable.

(d) Every weak-measurable function $f: I \rightarrow K$ is weak-equivalent to a weakly measurable function $g: I \rightarrow \overline{c o}^{*}(K)$.

(e ) For every bounded linear operator $T: C(K) \rightarrow L_{\infty}$, the operator $i^{\circ} T^{\circ} e$ is compact.

(f) For every weak*-measurable function $f: I \rightarrow K$, the operator $T_{f}$ is compact.

(g) For every weak*-measurable function $f: I \rightarrow K$, it holds that

$$
\inf _{n \geqq 1}\left\|T_{f}^{*}\left(r_{n}\right)\right\|=0 .
$$

(h) For every weak ${ }^{*}$-measurable function $f: I \rightarrow K$, the set $T_{f}^{*}\left(\Delta_{I}\right)$ contains no $\delta$-Rademacher tree.

Remark 2. In [1], Bator has proved : Let $K$ be a weak*-compact absolutely convex subset of $X^{*}$. If $K$ is not a weak Radon-Nikodym set, then there exists a DP-subset of $K$ which is not relatively norm compact. By Corollary 1, we can give a more concrete and refined form of this result as follows : Let $K$ be a weak ${ }^{*}$-compact subset of $X^{*}$. If $K$ is not a Pettis set, then there exists a weak ${ }^{*}$-measurable function $h: I \rightarrow K$ such that $T_{h}^{*}\left(B\left(L_{\infty}\right)\right)$ is a DP-subset of $\overline{a c o}^{*}(K)$ (: the weak*-closed absolutely convex hull of $K$ ) which is not relatively norm compact.

In Corollary 1, assume further that $K$ is convex. Then various characterizations of weak Radon-Nikodym sets can be obtained as follows. 
Corollary 2. Let $K$ be a weak*-compact convex subset of $X^{*}$. Then the following statements about $K$ are equivalent.

(a) The set $K$ is a weak Radon-Nikodym set.

(b) The set $K$ has the scalar point of continuity property.

(c). Every nonempty subset of $K$ is weak ${ }^{*}$-scalarly dentable.

(d) Every weak-measurable function $f: I \rightarrow K$ is weak-equivalent to a weakly measurable function $g: I \rightarrow K$.

(e ) For every bounded linear operator $T: C(K) \rightarrow L_{\infty}$, the operator $i \circ T \circ e$ is compact.

(f) For every bounded linear operator $T: L_{1} \rightarrow X^{*}$ such that $T\left(\Delta_{I}\right) \subset K$, the operator $T$ is Dunford-Pettis.

( $\mathrm{g}) \quad$ For every bounded linear operator $T: L_{1} \rightarrow X^{*}$ such that $T\left(\Delta_{I}\right) \subset K$, it holds that

$$
\inf _{n \geqq 1}\left\|T\left(r_{n}\right)\right\|=0
$$

( h ) The set $K$ contains no $\delta$-Rademacher tree.

Proof. The points to be noted are in the following.

(i) (b) $\Rightarrow$ (c). Suppose that (c) fails. Then there is a bounded subset $L$ of $K$ such that $L$ is not weak*-scalarly dentable. Then it easily follows that $M\left(=\overline{c o}^{*}(L) \subset K\right)$ is not weak*-scalarly dentable, since $S(x, \alpha, M) \supset S(x, \alpha$, L) for $x \in X$ and $\alpha>0$. Hence the same argument as in the proof of Theorem indicates that (b) fails.

(ii) $\quad$ (c) $\Rightarrow$ (d). This immediately follows, since $\overline{c o}^{*}\left(T_{f}^{*}\left(\Delta_{E}\right)\right) \subset K$ for every weak*-measurable function $f: I \rightarrow K$ and every $E \in \Lambda^{+}$.

(iii) (h) $\Rightarrow$ (a). Suppose that (a) fails. Then there is a weak*measurable function $h: I \rightarrow K$ such that $T_{h} *\left(\Delta_{I}\right)$ contains a $\delta$-Rademacher tree. As $T_{h}{ }^{*}\left(\Delta_{I}\right) \subset K$ by the separation theorem, $K$ contains a $\delta$-Rademacher tree and so (h) fails.

(iv) (a) $\Rightarrow(\mathrm{h})$. Suppose that (h) fails. Then the set $K$ contains a $\delta$-Rademacher tree $\left\{x^{*}(n, k): n=0,1, \cdots ; k=1, \cdots, 2^{n}\right\}$. Then, by the same argument as in the proof of Theorem, we have a weak*-measurable function $f: I$ $\rightarrow K$ such that $T_{f}^{*}\left(\Delta_{I}\right) \supset\left\{x^{*}(n, k): n=0,1, \cdots ; k=1, \cdots, 2^{n}\right\}$. This contradicts the statement (h) in Theorem, and so (a) fails.

$(\mathrm{v})$ The statement $(\mathrm{f})$ is equivalent to the following statement $\left(\mathrm{f}^{\prime}\right)$.

$\left(\mathrm{f}^{\prime}\right)$ For every weak*-measurable function $f: I \rightarrow K$, the operator $T_{f}$ is compact.

Indeed, assume that $\left(\mathrm{f}^{\prime}\right)$ is true and let $T: L_{1} \rightarrow X^{*}$ be a bounded linear operator that satisfies $T\left(\Delta_{I}\right) \subset K$. Then there exists a weak ${ }^{*}$-measurable function $f: I \rightarrow K$ such that 


$$
\left(x, T\left(\chi_{E}\right)\right)=\int_{E}(x, f(t)) d \lambda(t)
$$

for every $x \in X$ and every $E \in \Lambda$. Hence we get that

$$
\left(x, T_{f}^{*}\left(\chi_{E}\right)\right)=\left(T_{f}(x), \chi_{E}\right)=\int_{E}(x, f(t)) d \lambda(t)=\left(x, T\left(\chi_{E}\right)\right)
$$

for every $x \in X$ and every $E \in \Lambda$, whence $T_{f}^{*}\left(\chi_{E}\right)=T\left(\chi_{E}\right)$ for every $E \in \Lambda$. Since the operator $T_{f}^{*}$ is compact, $\left\{T\left(\chi_{E}\right): E \in \Lambda\right\}$ is relatively norm compact. So the operator $T$ is Dunford-Pettis.

Conversely, assume that (f) is true and let $f: I \rightarrow K$ be a weak ${ }^{*}$-measurable function. Then the operator $S_{f}: L_{1} \rightarrow X^{*}$ satisfies that $S_{f}\left(\Delta_{I}\right) \subset K$ by the separation theorem. Hence the set $\left\{S_{f}\left(\chi_{E}\right): E \in \Lambda\right\}$ is relatively norm compact. Thus the operator $T_{f}^{*}$ is compact and so is $T_{f}$.

By the same argument as this, it easily follows that the statement (g) is equivalent to the following statement $\left(\mathrm{g}^{\prime}\right)$.

$\left(\mathrm{g}^{\prime}\right)$ For every weak ${ }^{*}$-measurable function $f: I \rightarrow K$, it holds that

$$
\inf _{n \geqq 1}\left\|T_{f}^{*}\left(r_{n}\right)\right\|=0 .
$$

Consequently, we see that Corollary 2 holds.

In Theorem, assume further that $K$ is convex. Then we easily get various characterizations of localized weak precompactness as follows.

Corollary 3. Let $A$ be a bounded subset of $X$ and $K$ a weak-compact convex subset of $X^{*}$. Then the following statements about $A$ and $K$ are equivalent.

(a) The set $A$ is $K^{-}$weakly precompact.

(b) The set $K$ has the $\bar{A}^{*}-P C P$.

(c) Every nonempty subset of $K$ is weak ${ }^{*}-\bar{A}^{*}$-dentable.

(d) Every weak*-measurable function $f: I \rightarrow K$ is weak*-equivalent to a $\bar{A}^{*-}$ measurable function $g: I \rightarrow K$.

(e ) For every bounded linear operator $T: C(K) \rightarrow L_{\infty}$, the set $(i \circ T \circ e)(A)$ is relatively norm compact.

(f) For every weak*-measurable function $f: I \rightarrow K$, the set $T_{f}(A)$ is relatively norm compact.

(g) For every bounded linear operator $T: L_{1} \rightarrow X^{*}$ such that $T\left(\Delta_{I}\right) \subset K$, it holds that

$$
\inf _{n \geq 1}\left\{\sup _{x \in A}\left|\left(x, T\left(r_{n}\right)\right)\right|\right\}=0 .
$$

(h) The set $K$ contains no $A-\delta$-Rademacher tree. 
Finally, when $K=B\left(X^{*}\right)$ in Corollary 3, various characterizations of weakly precompact sets can be obtained as follows.

Corollary 4. Let $A$ be a bounded subset of $X$. Then the following statements about $A$ are equivalent.

(a) The set $A$ is weakly precompact.

(b) Every nonempty weak $k^{*}$ compact subset of $X^{*}$ has the $\bar{A}^{*}-P C P$.

(c) Every nonempty bounded subset of $X^{*}$ is weak $k^{*}-\bar{A}^{*}$-dentable.

(d) Every bounded weak*-measurable function $f: I \rightarrow X^{*}$ is weak ${ }^{*}$-equivalent to a bounded $\bar{A}^{*}$-measurable function $g: I \rightarrow X^{*}$.

( e ) For every bounded linear operator $T: C\left(B\left(X^{*}\right)\right) \rightarrow L_{\infty}$, the set $(i \circ T \circ e)(A)$ is relatively norm compact.

(f) For every bounded weak*-measurable function $f: I \rightarrow X^{*}$, the set $T_{f}(A)$ is relatively norm compact.

(g) For every bounded linear operator $T: L_{1} \rightarrow X^{*}$, it holds that

$$
\inf _{n \geqq 1}\left\{\sup _{x \in A}\left|\left(x, T\left(r_{n}\right)\right)\right|\right\}=0 .
$$

(h) Every nonempty bounded subset of $X^{*}$ contains no $A-\delta$-Rademacher tree.

Remark 3. In addendum of [18], Odell's result on weakly precompact sets has been stated as follows: The bounded subset $A$ of $X$ is weakly precompact if and only if for every Banach space $X^{\prime}$ and every Dunford-Pettis operator $T: X$ $\rightarrow X^{\prime}$, the set $T(A)$ is relatively norm compact. In virtue of Corollary 4, we can give a slight refinement of this result as follows. Let $A$ be a bounded subset of $X$. Then the following statements about $A$ are equivalent.

(a) The set $A$ is weakly precompact.

(b) For every Banach space $X^{\prime}$ and every Dunford-Pettis operator $T: X$ $\rightarrow X^{\prime}$, the set $T(A)$ is relatively norm compact.

(c) For every Dunford-Pettis operator $T: X \rightarrow L_{1}$, the set $T(A)$ is relatively norm compact.

(d) For every bounded weak ${ }^{*}$-measurable function $f: I \rightarrow X^{*}$, the set $T_{f}(A)$ is relatively norm compact.

Indeed, we have only to note that $T_{f}: X \rightarrow L_{1}$ is Dunford-Pettis for every bounded weak*-measurable function $f: I \rightarrow X^{*}$, which easily follows from the bounded convergence theorem.

\section{References}

[1] Bator, E. M., Remarks on completely continuous operators, Bull. Polish Acad. Sci. Math., 37 (1989), 409-413.

[2] Bator, E.M. and Lewis, P.W., Weak precompactness and the weak RNP, Bull. Polish Acad. Sci. 
Math., 37 (1989), 443-452.

[3] Fitzpatrick, S., Separably related sets and the Radon-Nikodym property, Illmois J. Math., 29 (1985), 229-247.

[4] Fremlin, D.H., Pointwise compact sets of measurable functions, Manuscripta Math., 15 (1975), 219-242.

[5] Ghoussoub, N., Godefroy, G., Maurey, B. and Schachermayer, W., Some topological and geometrical structures in Banach spaces, Mem. Amer. Math. Soc., Providence, 378 (1987).

[6] Girardi, M. and Uhl, Jr, J.J., Slices, RNP, strong regularity, and martingales, Bull. Austral. Math. Soc., 41 (1990), 411-415.

[7] Ionescu Tulcea, A. and Ionescu Tulcea, C., Topics in the theory of lifting, Ergeb. Math., Springer, 48 (1969).

[8] Matsuda, M., On Saab's characterizations of weak Radon-Nikodym sets, Publ. RIMS, Kyoto Univ., 22 (1985), 921-941.

[9] A characterization of weak Radon-Nikodym sets in dual Banach spaces, Publ. RIMS, Kyoto Univ., 23 (1986), 551-559.

[10] - A characterization of Pettis sets in dual Banach spaces, Publ. RIMS, Kyoto Univ., 27 (1991), 827-836.

[11] - A characterization of non-Pettis sets in terms of martingales, Math. Japon., 38 (1993), 177-183.

[12] - Remarks on Pettis sets, Rep. Fac. Sci. Shizuoka Univ., 28 (1994), 17-23.

[13] - A characterization of Pettis sets in terms of the Bourgain property, Math. Japon., 41 (1995), 433-439.

[14] Weak*-midpoint-Bocce-dentability and Pettis sets, preprint.

[15] Odell, E. and Rosenthal H., A double-dual characterization of separable Banach spaces containing $l_{1}$, Israel J. Math., 20 (1975), 375-384.

[16] Riddle., L. H. and Saab, E., On functions that are universally Pettis integrable, Illinois J. Math., 29 (1985), 509-531.

[17] Rosenthal, H., A characterization of Banach spaces containing $l_{1}$, Proc. Nat. Acad. Sci., 71 (1974), 2411-2413.

[18] . Pointwise compact subsets of the first Baire class, Amer. J. Math., 99 (1977). 362-378.

[19] Some recent discoveries in the isomorphic theory of Banach spaces, Bull. Amer. Math. Soc., 84 (1978), 803-831.

[20] Saab, E., Some characterizations of weak Radon-Nikodym sets, Proc. Amer. Math. Soc., 86 (1982), 307-311.

[21] Saab, E. and Saab, P., A dual geometric characterization of Banach spaces not containing $l_{1}$, Pacif. J. Math., 105 (1983), 415-425.

[22] Talagrand, M., Pettis integral and measure theory, Mem. Amer. Math. Soc., Providence, 307 (1984). 
